\title{
Eight Weeks Sit-Up versus Isometric Abdominal Training: Effects on Abdominal Muscles Strength Capacity
}

\author{
Christoph Anders* (D), Franz Ludwig, Florian Sänger and Martin Marks \\ Clinic for Trauma, Hand and Reconstructive Surgery, Division of Motor Research, Pathophysiology and Biomechanics, \\ Jena University Hospital, Germany
}

\begin{abstract}
Background: The importance of abdominal muscles to achieve and maintain adequate mobility and stability of the trunk has long been underestimated. We therefore examined different abdominal muscles training modalities (classical sit-ups vs. isometric training) with regard to their effects on trunk muscle's strength performance.

Methods: A total of 79 healthy persons aged 19-37 years were examined (sit-up group: $n=19$, male, isometric group: $n$ = 60; 31 female, 29 male). All participants completed an eight weeks submaximal abdominal muscles training program of either sit-ups or a supine isometric task. Investigations took place twice: Just before (T1) and after completion of the training (T2). Each training program started at $50 \%$ of the number of repetitions or the respective maximum holding time that were systematically increased over time. Maximum test performance and maximum isometric contraction forces (MVC) in flexion and extension directions were measured before and after the training.
\end{abstract}

Results: At T2 the number of sit-ups increased about $147 \%+97 \%$, whereas the maximum isometric holding times increased about $55 \% \pm 56 \%$ (females) and $54 \%+69 \%$ (males). A significant increase of the flexion-MVC could be detected for the isometric training group (female: $9.8 \pm 10.2$; male: $6.3 \pm 8.6$ ), but the sit-up group failed to show any systematic change. All MVC force levels were larger for extension than for flexion direction and were higher for males in comparison with females. Interestingly, during extension, when normalized to the upper body weight, the observed gender differences disappeared in the isometric training group.

Conclusions: An eight weeks submaximal isometric abdominal training is suited to significantly increase the MVC levels for flexion, but sit-up training is not. Gender- related differences in MVC levels are to be treated with caution, since if MVC levels are normalized according to anthropometric data these differences disappear, at least for the extension.

\section{Keywords}

Trunk muscle training, Sit-up training, Isometric training, Trunk muscle MVC

\section{Introduction}

Adequate physical fitness is an essential prerequisite for withstanding physical daily burdens while ensuring the necessary trunk stability. With regard to the trunk muscles, any training of the abdominal muscles appears to be particularly important. Although abdominal muscles are significantly weaker than the back muscles, their importance for achieving sufficient trunk stability is undisputed [1-4]. Their performance can be defined in two ways: Endurance capacity and maximum strength capacity. Since abdominal muscles fiber composition shows a significantly higher Type II fiber proportion compared to the back muscles, their endurance training appears to be particularly important because their fiber composition makes them more difficult to train $[5,6]$. The question of which training exercise and with which training modality scores best is subject of an ongoing discussion [7-10]. Furthermore, it is unclear to what extent a defined strength-endurance training affects their maximum strength capacity.
A very popular and easy to perform exercise, would first be the sit-up in its classical execution mode with hip and knee joints bent $90^{\circ}$ [8]. Besides the abdominal target muscles, the deep hip flexors, i.e. the iliopsoas muscles, are also activated during sit-ups [11]. Whether execution variations lead to different results is still subject of investigations [10]. A completely different training strategy is pursued by the core sta-

*Corresponding author: Christoph Anders, Clinic for Trauma, Hand and Reconstructive Surgery, Division of Motor Research, Pathophysiology and Biomechanics, Jena University Hospital, 07740 Jena, Germany, Tel: 0049-3641-932-8960, Fax: 00493641-932-8967

Accepted: June 25, 2020

Published online: June 27, 2020

Citation: Anders C, Ludwig F, Sänger F, et al. (2020) Eight Weeks Sit-Up versus Isometric Abdominal Training: Effects on Abdominal Muscles Strength Capacity. Arch Sports Med 4(1):198-204 
Citation: Anders C, Ludwig F, Sänger F, et al. (2020) Eight Weeks Sit-Up versus Isometric Abdominal Training: Effects on Abdominal Muscles Strength Capacity. Arch Sports Med 4(1):198-204

Table 1: Anthropometric data of study participants at initial investigation.

\begin{tabular}{|l|l|l|l|}
\hline & Situp (male $\mathbf{n}=19)$ & Isometric (female, $\mathbf{n}=\mathbf{3 1})$ & Isometric $($ male, $\mathbf{n}=\mathbf{2 9})$ \\
\hline Age $[$ years] & $23.1 \pm 2.9$ & $23.2 \pm 2.9$ & $27.8 \pm 5.4^{\S \varsigma}$ \\
\hline Height $[\mathrm{cm}]$ & $179.8 \pm 6.4$ & $168.8 \pm 5.4^{\S}$ & $179.6 \pm 6.1^{\S}$ \\
\hline Weight $[\mathrm{kg}]$ & $71.5 \pm 6.8$ & $64.5 \pm 9.8^{\S}$ & $77.9 \pm 8.9^{\S \varsigma}$ \\
\hline BMI $\left[\mathrm{kg} / \mathrm{m}^{2}\right]$ & $22.1 \pm 2.0$ & $22.7 \pm 3.3$ & $24.2 \pm 2.6^{\S}$ \\
\hline UBT $[\mathrm{Nm}]$ & $106.3 \pm 13.8$ & $84.7 \pm 14.1^{\S}$ & $114.9 \pm 16.2^{\S}$ \\
\hline
\end{tabular}

BMI: Body mass index; data are given as means \pm SD; ${ }^{\S} p<0.05$ vs. Sit-up; ${ }^{\$} p<0.05$ vs. isometric, female

bility training, which uses isometric tension exercises [12-14]. This training is mainly used for the targeted improvement of core stability and therefore not primarily aims at an increase in strength [15].

However, own findings also suggest other correlations, since we were able to prove that the nature of force control has a considerable influence on the SEMG amplitude level, i.e. the strain level of abdominal muscles [16]. We could show that for all abdominal muscles the effort level during postural submaximal isometric tension was higher than during load-identical force-controlled situations. The measured EMG amplitudes even reached levels exceeding those obtained during maximum isometric force exertion. Since these findings were collected from untrained persons, the question arose whether these differences were due to the lack of experience with the tested exercise or if they had to be considered as a specific feature.

For this reason, two studies were conducted to test if a specific sit-up training or an isometric training of the abdominal muscles would have effects on the maximum force capacity of abdominal muscles.

\section{Method}

A total of 79 healthy persons aged 19-37 years were examined. Details of the study population are given in Table 1.

All participants were informed about the purpose and procedure of the study and gave their written consent to participate. The local ethics committee (4348-02/15) approved the study.

To exclude relevant orthopedic and neurologic disorders, participants were clinically investigated and further queried about their medical history. Besides general health problems possibly interfering with unrestricted study participation, specific orthopedic exclusion criteria were spine surgery, any actual pain, or pain during any of the tested exercises.

\section{Investigation}

After Surface-EMG instrumentation (SEMG, see below) participants performed isometric maximum voluntary contraction (MVC) tests in flexion and extension directions.

This was performed in a computerized test and training device (CTT Centaur, BfMC, Germany). In the device, the subject's lower body up to the iliac crest was fixed while the upper body maintained its freedom of movement (Figure 1).

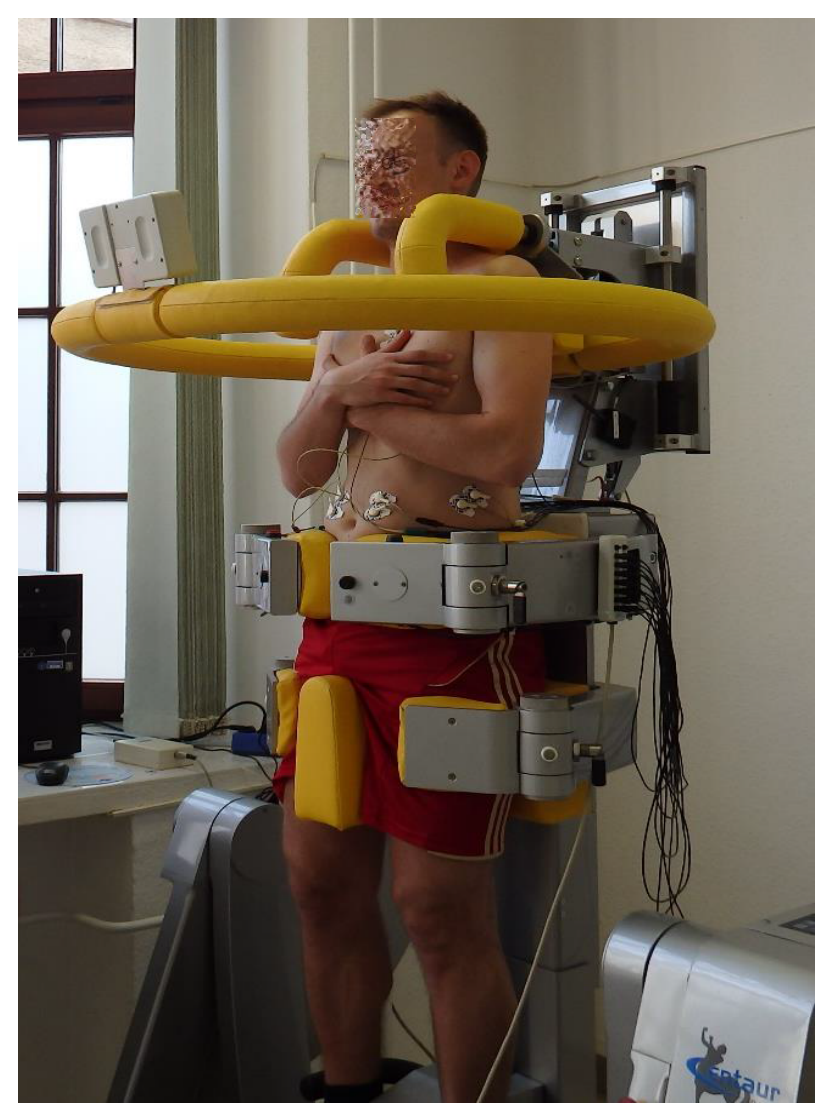

Figure 1: Example of a subject performing an MVC trial in the device. Please note that the subject is positioned upright with his lower body fixed and the upper body remaining freedom of movement to push against the harness.

The device was equipped with an open harness positioned over the subject's shoulders. Strain gauges together with a crosshair display, visible to the subjects, provided biofeedback during the MVC tasks. MVC tasks were performed in upright position in flexion and extension directions and were repeated two times with the highest value serving as the true MVC value. Detailed explanation of the procedure and verbal encouragement was provided for every volunteer $[17,18]$.

Additionally, the upper body weight was determined. This was done by tilting the device to $90^{\circ}$ and reading out the corresponding force values. Care was taken to ensure that there were no residual contractions of the back extensors to avoid incorrect values. Again, the highest value out of three repetitions was used. To calculate the upper body torque (UBT) we determined the distance between the line between the two 
a)

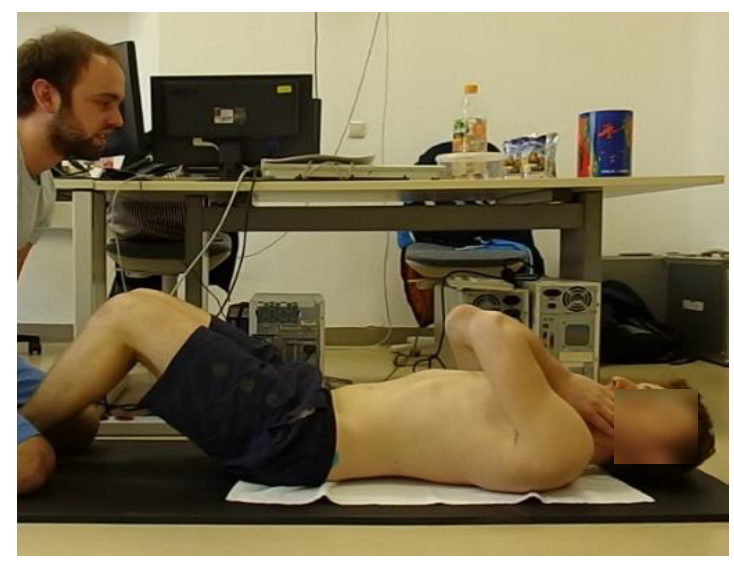

b)

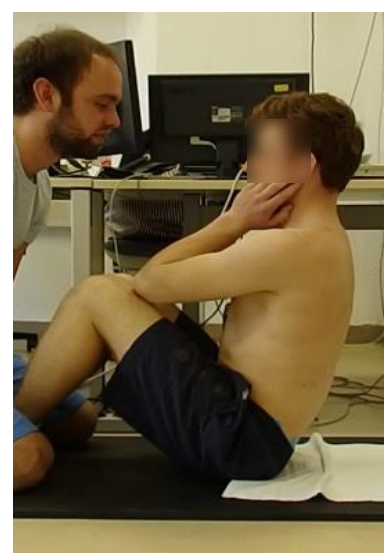

C)

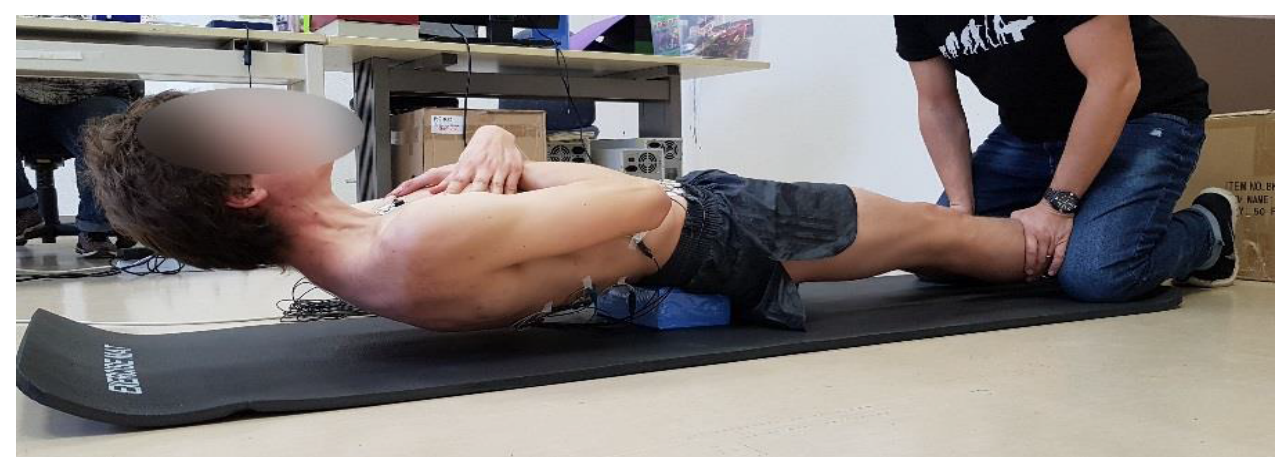

Figure 2: Exercise execution for the two training modes. Upper row: Sit-up execution, a) Starting position; b) End position. Execution time was one second per movement phase; c) Isometric training position.

scapular spines and the iliac crest as the length of the upper body segment.

\section{Performance tests and training}

At first visit (T1) all participants performed a maximum situp or hold test, respectively.

For the sit-up test, legs were positioned with the hip and knee joints both flexed $90^{\circ}$ and feet fixed in position (Figure 2). Arms were kept sidewise the head. To perform the sit-ups, participants were asked to raise their upper body within one second until the elbows touched the thighs and then to lower their upper body, again within one second. The next sit-up took place immediately after their shoulder blades touched the floor. The test was performed until subjects couldn't continue [10].

Subjects were asked to conduct the sit-up training the same way as for the maximum performance test, once a day. During the first week the required number of sit-ups was $50 \%$ of the maximum number of repetitions at T1. After seven days, the target number of repetitions increased by $10 \%$ of the maximum number of repetitions at T1, which was again performed for one week. This increase regime was continued for the whole training period of eight weeks. At follow-up (T2) another maximum performance sit-up test was conducted.
For the isometric holding test participants positioned themselves in an outstretched supine position with a yoga brick placed under the sacrum and the arms crossed over the chest. With fixed straight legs they were then asked to lift their upper body off the floor until horizontal position and retain this position until they were unable to maintain the desired posture (Figure 2).

The isometric training was also performed once a day. Initial training intensity was $50 \%$ of the maximum holding time, similar to the procedure for the sit-up training. On day seven, however, subjects performed another maximum holding test on their own. Fifty percent of this maximum holding time then served as the adapted training time for the following week. This adaptive training regime was again continued for 8 weeks.

All study participants maintained a training diary. To avoid interferences with physical activities other than normal intensity, participants were asked to avoid physical activities that are particularly strenuous or require a lot of stamina.

The respective maximum repetition numbers or holding times together with the results of the isometric MVC tests served as the outcome variables. With respect to the MVC tests, the achieved force values in $\mathrm{N}$, the calculated torque values in $\mathrm{Nm}$, and also the upper body torque ratio (UBTR), 


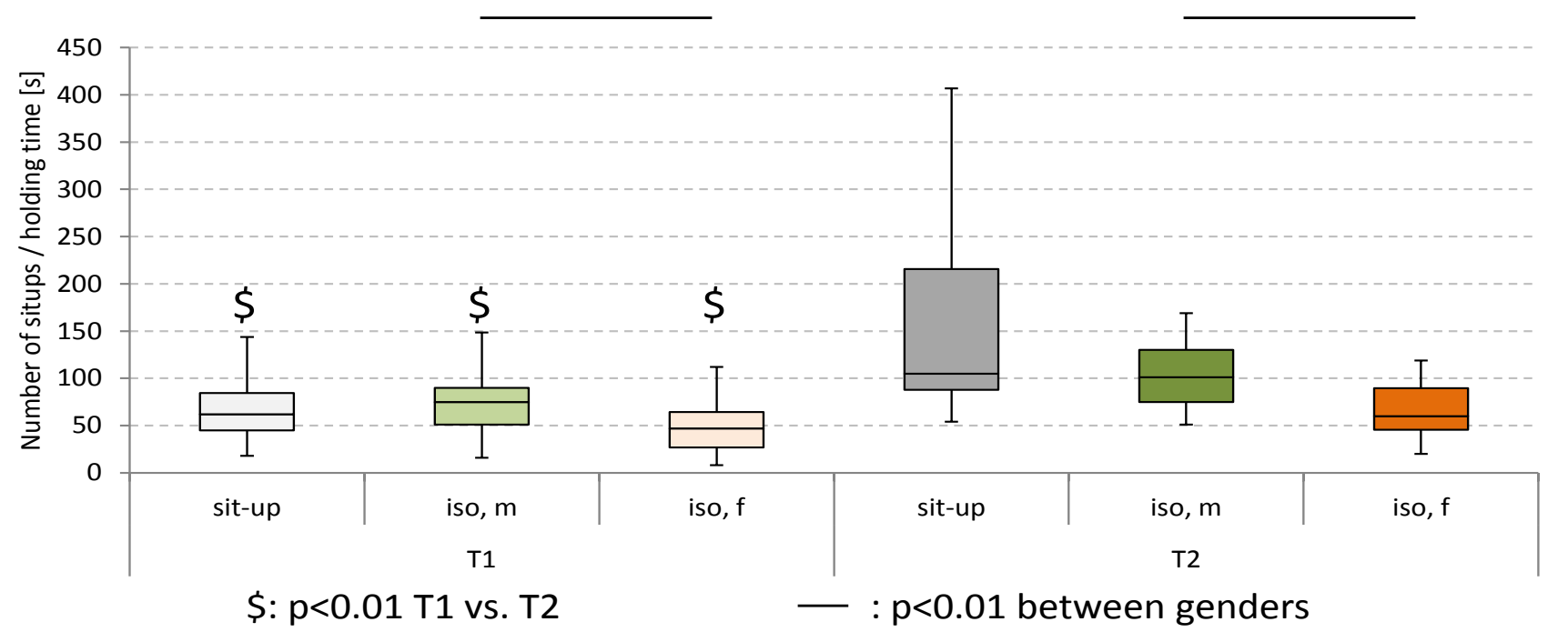

Figure 3: Results of the maximum performance tests for both, the sit-up repetition number and the isometric holding time before (T1) and after the training period (T2). Results of the statistical calculation for between times and between genders (isometric training only) are provided ( $p$-values are Bonferroni-corrected).

Sit-up: Sit-up training; Iso: Isometric abdominal training.

i.e. the ratio of maximum force relative to the upper body weight, were evaluated. A repeated measures ANOVA with "Time" serving as the within subject factor (2 classes: T1, T2) and "Training" (2 classes: Sit-up, isometric) together with "Gender" ( 2 classes: Female, male) as the between subject factor (saturated model). Post-hoc analyses considered temporal comparisons, i.e. the training related effects, as well as comparisons between the training modalities and comparisons between genders for the isometric training group.

\section{Results}

Both, the maximum number of sit-ups (increase $147 \% \pm$ $97 \%$, Figure 3 ) and the holding time (increase female $55 \% \pm$ $56 \%$, male $54 \% \pm 69 \%$ ) significantly increased from T1 to T2. In the isometric training group, regardless of investigation time men always reached significantly longer holding times as women (Figure 3).

In the ANOVA for the main factor "Time" all MVC parameters reached highly significant $p$-values for flexion (all $p$-values $<0.001$ ), but not for extension (all p-values $>0.780$ ). The Main factor "Gender" showed highly significant differences as well ( $p<0.001)$, except for the UBTR values during extension ( $p=0.762$ ). In contrast, "Training" reached significant $p$-values for extension but not for flexion (all p-values > 0.245).

In detail, the MVC force values and torques were always higher for the men than for the women, regardless of the investigation time of (Figure 3 ), and generally higher for extension than for flexion (all $p<0.001$, effect sizes 0.878-3.431). However, only for the isometric training group an increase in flexion MVC could be demonstrated (Figure 4). The MVC force values and the torques for extension significantly decreased from T1 to T2 for the women of the isometric training group, while for the men in this training group a significant increase in extension MVC was demonstrated over time.
For the UBTR, men continued to show higher values for flexion than women, but for extension no difference between genders in the isometric training group could be demonstrated, whereby the UBTR values for the sit-up group were always higher than those of the holding group. The only training-related difference for the UBTR was a significant increase in flexion direction for the women of the isometric training group.

\section{Discussion}

We could clearly show that an eight weeks specific training could effectively increase the performance for the trained exercise, respectively. However, transfer effects towards an increase in MVC values of the abdominal muscles could only be proven for the isometric training group. MVC values of the back muscles showed unsystematic training effects: While no changes were detectable in the sit-up group, the men in the isometric training group showed a noticeable increase in strength and torque values, whereas a reduction was detectable for the female participants. In accordance with the known gender differences, both the performance tests and the MVC values showed gender differences with higher values for the men, as well as the expected differences in force values between flexion and extension, i.e. between abdominal and back muscles for the isometric MVC tests. Interestingly, when normalized to upper body weight the gender differences for extension MVC values disappeared.

For the performance tests, both training modes showed significant improvements, but improvement dimensions differed largely. This was difficult to explain on first sight. While the sit-up group showed an average increase of $147 \%$, the improvements for the holding time were far behind with $55 \%$ (females) and 54\% (males), respectively. Besides these considerable differences, it is interesting to note that the relative increases for men and women in the holding test were 


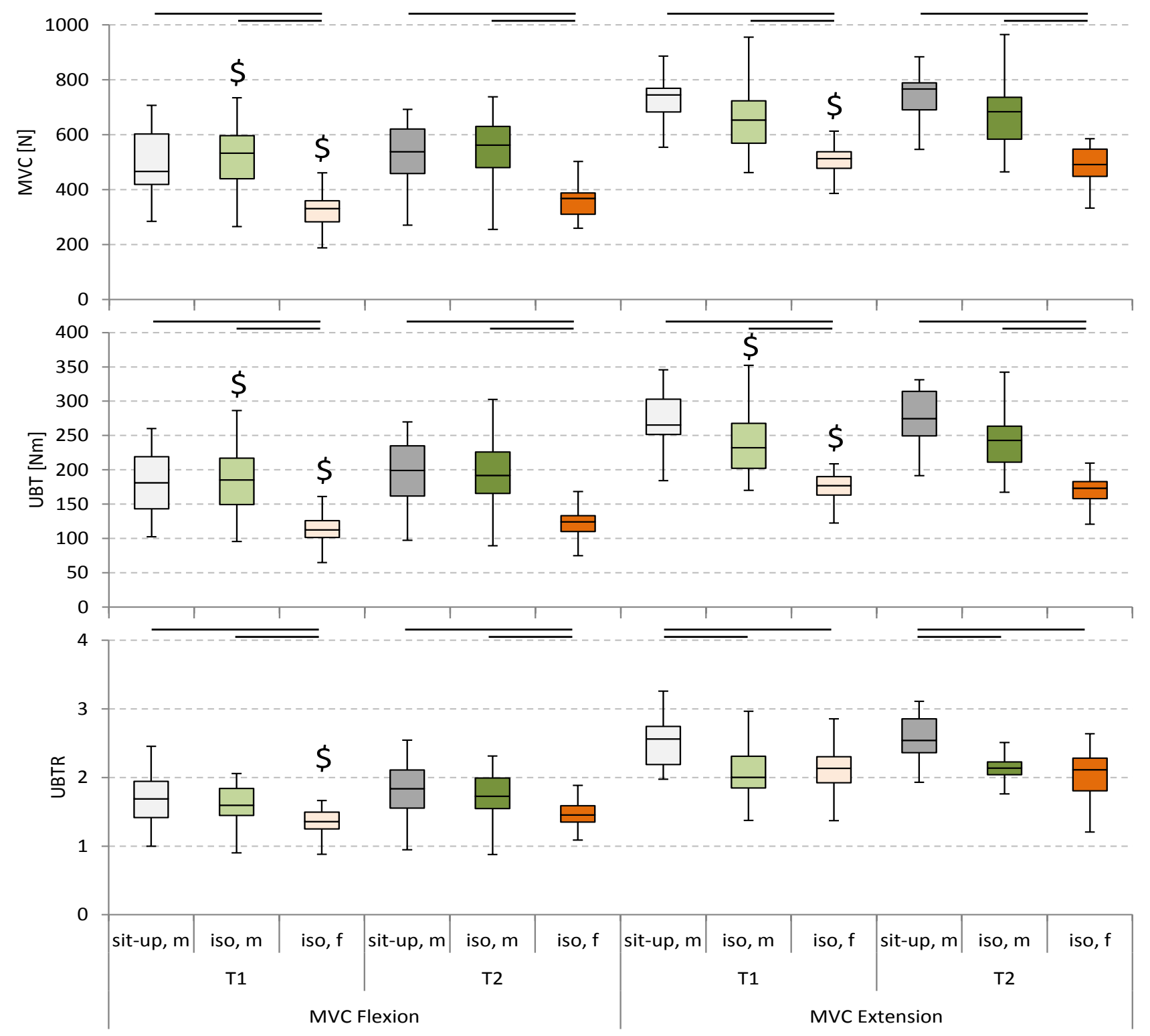

\$: $p<0.05$ T1 vs. T2

- $p<0.01$ between training groups /genders

Figure 4: Results for the Maximum force test for trunk flexion and extension before (T1) and after the training period (T2), separately for the different training modes and genders. Upper row: Maximum voluntary contraction (MVC) values in N, Middle row: Maximum Upper body torque (UBT) values in Nm, lower row: Upper body torque ratio (UBTR) values (ratio between upper body weight and MVC levels). Results of the statistical calculation for between times and between training group or genders (isometric training only) are provided ( $p$-values are Bonferroni-corrected).

Sit-up: Sit-up training; iso: Isometric abdominal training.

virtually identical. One reason for the observed differences could be that the training regime for the sit-up group, starting from the initial test, prescribed a fixed rate of increase of $10 \%$ of the initial value each week. Thus, during the eight weeks of training, there was a seven-fold increase of $10 \%$ each time. According to this, the number of training repetitions at the time of the follow-up examination was already $120 \%$ of the initial value: In other words $20 \%$ more than had been achieved during the initial maximum performance test. In contrast, the training regime for the isometric training group included an adaptive progression algorithm. Here, the individual motivational aspects play an important role to accom- plish the weekly maximum test under home conditions. However, the individual adaptive control of the isometric training was deliberately chosen to prevent possible overloading and thus training interruption. Furthermore, there is another essential difference between the two training modalities: While sit-ups are a dynamic training and thus the load intensity changes constantly during the execution, the upper body was always statically loaded with the complete upper body weight during the isometric training exercise. Due to this permanent isometric load, blood supply of the abdominal muscles is less favorable, resulting in more pronounced muscular fatigue if compared with a dynamic load situation. 
For the static endurance capacity of the trunk muscles there is sufficient data for the back muscles [19-23], but the data for the abdominal muscles is clearly worse. We found only exemplary studies for the isometric endurance test of the abdominal muscles, but in variation to our study design, these were performed in $60^{\circ}$ flexion position, i.e. with $50 \%$ of the upper body weight and flexed hip joints. For this, depending on the examination, very different values of approx. $145 \mathrm{~s}$ [21] and approx. $350 \mathrm{~s}$ were found [20]. Although for the study by Evans, et al. [20] the holding times were partly analyzed gender-specific, no systematic differences could be found. This was not the case in the present study - here the holding times of women were only about $65 \%$ of the times reached by the men, regardless of investigation time.

However, the different exercise performance in the holding test compared to the data already published has twofold reasons. At first the actual study applied double the load in comparison with the flexed position. At second, the deep hip flexors, i.e. the psoas muscles and also the muscles of the thigh were actively involved in the exercise performance in the case of the angled upper body position. This additional activation could be avoided with high certainty by applying the test in an out stretched body position. Thus, to execute the isometric exercise participants had to rely on their abdominal muscles almost completely. The abdominal muscles, due to their higher functional Type-II fiber cross-sectional area [5] are more prone to fatigue than the already stronger back muscles, that further show a larger proportion of Type-I fibers [24-26]. Further, the available cross-sectional area of the Type II fibres is significantly smaller in women than in men [26]. Therefore, the observed gender-related differences can be explained by taking into account the high muscular strain level. With respect to own previous investigations it can be assumed that the stress on the abdominal muscles during the holding test was close to or even above the values during the MVC test [16].

An effect of the eight-weeks training on maximum strength performance was only observed for the isometric training, although the relative increase of $6 \%$ for men and $10 \%$ for women seems rather sobering in light of the eight-weeks training period. However, no systematic change in MVC values could be demonstrated for the sit-up training group. Therefore, an eight-week sit-up training is not suitable to systematically influence the maximum strength of the abdominal muscles. For the isometric MVC during extension, which was also tested, a significant, albeit small, decrease in strength values was observed for the women in the isometric training group (decrease of $3.1 \%$, values not shown). In contrast, the men of the isometric training group showed a slight but non-significant increase of $3 \%$ in their extension MVC values, which was even significantly detectable in the UBT values. These inhomogeneous results for the same training modality are difficult to interpret and require further systematic investigations.

As expected, the women showed significantly and relevantly lower values than the men for both, the force values and the upper body torques but also for the ratio between MVC values and upper body weight, the UBTR $[27,28]$. However, it is interesting that these differences could not be sys- tematically detected for the UBTR during extension. For this, the sit-up group already proved to be superior to the isometric training group at T1. The superiority over the men of the isometric training group can possibly be explained by the fact that the sit-up group was about five years younger [29]. In addition, their UBT also tended to be lower than that of the males of the isometric training group. It is particularly noteworthy, however, that for the UBTR values, at least in the isometric training group, no systematic differences occurred between the values of males and females. Since the women had significantly lower upper body torques and thus also upper body weights, the clear gender differences of the MVC force or torque values were thus lost. Thus, the examined women had just as much power reserve for the movement and stabilization of their upper bodies as the men. This result highlights the importance of assessing force performance data not only as absolute values but also as normalized values that take into account the individual anthropometric situation.

\section{Conclusions}

As expected, an eight-weeks training of the abdominal muscles results in an improved performance of the trained exercise. The performance gains for a dynamic sit-up training are significantly larger than during an isometric training. However, only the isometric abdominal muscles training was suited to improve the isometric maximum force capacity of the abdominal muscles.

\section{Declarations of Interest}

None.

\section{References}

1. Cresswell AG, Oddsson L, Thorstensson A (1994) The influence of sudden perturbations on trunk muscle activity and intra-abdominal pressure while standing. Exp Brain Res 98: 336-341.

2. O'Sullivan PB, Phyty GD, Twomey LT, et al. (1997) Evaluation of specific stabilizing exercise in the treatment of chronic low back pain with radiologic diagnosis of spondylolysis or spondylolisthesis. Spine (Phila Pa 1976) 22: 2959-2967.

3. Puntumetakul R, Areeudomwong P, Emasithi A, et al. (2013) Effect of 10-week core stabilization exercise training and detraining on pain-related outcomes in patients with clinical lumbar instability. Patient Prefer Adherence 7: 1189-1199.

4. Tesh KM, Dunn JS, Evans JH (1987) The abdominal muscles and vertebral stability. Spine (Phila Pa 1976) 12: 501-508.

5. Haggmark T, Thorstensson A (1979) Fibre types in human abdominal muscles. Acta Physiol Scand 107: 319-325.

6. Thorstensson A, Carlson H (1987) Fibre types in human lumbar back muscles. Acta Physiol Scand 131: 195-202.

7. Andersson EA, Nilsson J, Ma Z, et al. (1997) Abdominal and hip flexor muscle activation during various training exercises. Eur $\mathrm{J}$ Appl Physiol Occup Physiol 75: 115-123.

8. Beim GM, Giraldo JL, Pincivero DM, et al. (1997) Abdominal strengthening exercises: A comparative EMG study. Journal of Sport Rehabilitation 6: 11-20.

9. Guimaraes A, Vaz MA, De Campos M, et al. (1991) The contribution of the rectus abdominis and rectus femoris in twelve select- 
ed abdominal exercises. An electromyographic study. J Sports Med Phys Fitness 31: 222-230.

10. Sullivan W, Gardin FA, Bellon CR, et al. (2015) Effect of traditional vs. modified bent-knee sit-up on abdominal and hip flexor muscle electromyographic activity. J Strength Cond Res 29 3472-3479.

11. Juker D, McGill S, Kropf P, et al. (1998) Quantitative intramuscular myoelectric activity of lumbar portions of psoas and the abdominal wall during a wide variety of tasks. Med Sci Sports Exerc 30: 301-310.

12. Borghuis J, Hof AL, Lemmink KA (2008) The importance of sensory-motor control in providing core stability: Implications for measurement and training. Sports Med 38: 893-916.

13. Hodges PW (2003) Core stability exercise in chronic low back pain. Orthop Clin North Am 34: 245-254.

14. Huxel Bliven KC, Anderson BE (2013) Core stability training for injury prevention. Sports Health 5: 514-522.

15. Waldhelm A, Li L (2012) Endurance tests are the most reliable core stability related measurements. Journal of Sport and Health Science 1: 121-128.

16. Hansen L, Anders C (2014) Influence of different control strategies on muscle activation patterns in trunk muscles. Physiol Rep 2: e12229.

17. Argus CK, Gill ND, Keogh JWL, et al. (2011) Acute effects of verbal feedback on upper-body performance in elite athletes. J Strength Cond Res 25: 3282-3287.

18. McNair PJ, Depledge J, Brettkelly M, et al. (1996) Verbal encouragement: Effects on maximum effort voluntary muscle action. $\mathrm{Br}$ J Sports Med 30: 243-245.

19. Biering Sorensen F (1984) Physical measurements as risk indicators for low-back trouble over a one-year period. Spine (Phila Pa 1976) 9: 106-119.
20. Evans K, Refshauge KM, Adams R (2007) Trunk muscle endurance tests: Reliability, and gender differences in athletes. J Sci Med Sport 10: 447-455.

21. McGill SM, Childs A, Liebenson C (1999) Endurance times for low back stabilization exercises: Clinical targets for testing and training from a normal database. Arch Phys Med Rehabil 80: 941-944.

22. Stewart M, Latimer J, Jamieson M (2003) Back extensor muscle endurance test scores in coal miners in Australia. J Occup Rehabil 13: 79-89.

23. Tsuboi H, Nishimura Y, Sakata T, et al. (2013) Age-related sex differences in erector spinae muscle endurance using surface electromyographic power spectral analysis in healthy humans. Spine J 13: 1928-1933.

24. Elder GC, Bradbury K, Roberts R (1982) Variability of fiber type distributions within human muscles. J Appl Physiol Respir Environ Exerc Physiol 53: 1473-1480.

25. Kalimo H, Rantanen J, Viljanen T, et al. (1989) Lumbar muscles: Structure and function. Ann Med 21: 353-359.

26. Mannion AF, Dumas GA, Cooper RG, et al. (1997) Muscle fiber size and type distribution in thoracic and lumbar regions of erector spinae in healthy subjects without low back pain: Normal values and sex differences. J Anat 190: 505-513.

27. Anders C, Huebner A, Faenger B (2018) The neuromuscular efficiency of trunk muscles is altered by age in the abdominal muscles but by gender in the back muscles. Int J Sports Exerc Med 4: 089.

28. Huebner A, Faenger B, Scholle HC, et al. (2015) Re-evaluation of the amplitude-force relationship of trunk muscles. J Biomech 48: 1198-1205.

29. Adedoyin RA, Mbada CE, Farotimi AO, et al. (2011) Endurance of low back musculature: Normative data for adults. J Back Musculoskelet Rehabil 24: 101-109. 